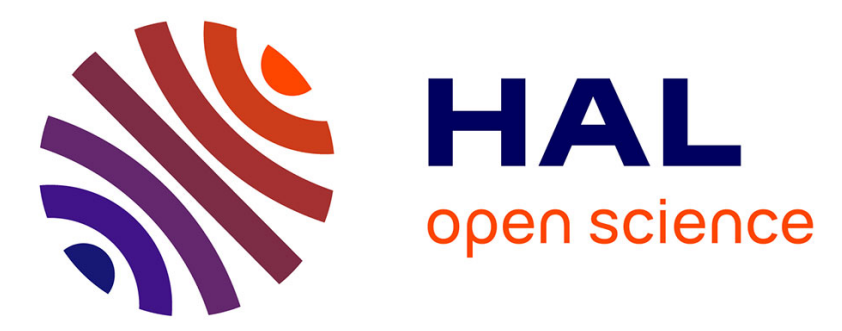

\title{
Acoustic emission from successive impacts on elastic membranes: The physics of the screaming balloon
}

M. Hibat Allah, B. Isnard, N. Taberlet, J. Ferrand, M Hibat, Nicolas Plihon

\section{To cite this version:}

M. Hibat Allah, B. Isnard, N. Taberlet, J. Ferrand, M Hibat, et al.. Acoustic emission from successive impacts on elastic membranes: The physics of the screaming balloon. EPL - Europhysics Letters, 2019, 126 (6), pp.64001. 10.1209/0295-5075/126/64001 . hal-02398896

\section{HAL Id: hal-02398896 https://hal.science/hal-02398896}

Submitted on 11 Dec 2019

HAL is a multi-disciplinary open access archive for the deposit and dissemination of scientific research documents, whether they are published or not. The documents may come from teaching and research institutions in France or abroad, or from public or private research centers.
L'archive ouverte pluridisciplinaire HAL, est destinée au dépôt et à la diffusion de documents scientifiques de niveau recherche, publiés ou non, émanant des établissements d'enseignement et de recherche français ou étrangers, des laboratoires publics ou privés. 


\title{
Acoustic emission from successive impacts on elastic membranes: the physics of the screaming balloon
}

\author{
N. Taberlet ${ }^{1}$, J. Ferrand ${ }^{1}$, M. Hibat Allah ${ }^{1}$, B. Isnard ${ }^{1}$ and N. Plihon ${ }^{1}$ \\ 1 Univ Lyon, Ens de Lyon, Univ Claude Bernard, CNRS, Laboratoire de Physique, Département de Physique, F-69342 \\ Lyon, France
}

\author{
PACS 46.40.-f - Vibrations and mechanical waves \\ PACS 43.20.+g - General linear acoustics \\ PACS 46.25.-y - Static elasticity
}

\begin{abstract}
When a hex nut or a ridged-edge coin, placed inside an inflated rubber balloon and spun vigorously, it emits a surprisingly loud and clear sound as the spinning object impacts the rubber and triggers vibrations of the membrane, a phenomenon known as the screaming balloon. We identify the mechanisms behind the acoustic emission and show that the fundamental frequency of the sound is given solely by the rate of successive impacts of the spinning object onto the membrane as it rolls without slipping. A counter-intuitive observation is that the acoustic power emitted by a given ridged-edge object remains independent of the size of the balloon (over a wide range of volume) in which it spins. This experimental finding is explained by the influence of the tension within the membrane on the acoustic intensity. Finally, we propose a scaling law for the frequency-dependence of the acoustic intensity and show that the sound level depends greatly on the number of ridges on the edge of the spinning object.
\end{abstract}

Introduction. - The physics of musical instruments is a rich and fascinating subject involving complex and highly coupled physical phenomena [1]. Linear theories only constitute a first-order model [2] as a complete understanding of most instruments require to include non-linear effects (e.g. the interplay between stick-slip and the natural modes of vibration of a bowed string $[3,4]$, the exact design of the bell of a trumpet [5], or the equal temperament of a piano [6], the trapping of acoustic waves in musical saws due to the bending of the metal strip $[7,8]$ ). Bidimensional geometries such as drums exhibit sophisticated normal modes whose frequencies are not commensurable (as in Chladni figures [9]), producing a sound sometimes regarded as dissonant. However, by locally adjusting the thickness (or the mass per unit surface) of a membrane, on may turn tabla drums are turned into harmonic instruments [10]. In the singing wine glasses [11-13] as well as in the tibetan singing bowl [14], sound emission emerges from the coupling between the mechanical resonances of tri-dimensional structures and fluid dynamics. In all cases the frequency of the acoustic emission is determined by the resonant eigenfrequencies of strings, tubes, plates, or tridimensional structures - the sound level being frequently amplified through a sound board, a sound box or a bell.
Sound emission could also originate from repetitive pulses on structures. Natural examples include stridulations [15] where sound is created by insects rubbing their plectrum (or scraper) on the ridged pars stridens, as heard for cicadas or crickets [16]. A similar process is used for some percussion instruments, such as the guiro or the washboard where sound is generated by rubbing a stick (or nails) on a ridged wooden or metallic surface [17]: periodic pulses thus excite the structure and the periodicity is solely set by the velocity of the stick (or of the hand). From the musical point of view, the sound perception is related to the pitch. While for resonant percussions the pitch is given by the resonance features, it strongly depends upon the temporal properties of the impulses in the case of repetitive pulses [18]. In the case of periodic pulses at high audible frequencies - typically above one hundred Hertz - the pitch was shown to be unambiguously determined by the periodicity of the pulses $[19,20]$. In this article we investigate the sound produced by a hex nut (or a ridged-edge coin) spinning inside an inflated rubber balloon (see Fig.1 and Supplementary Video).

On the high-speed video (parts 2 and 4), one can see the edges of the nut protruding out of the balloon and setting the membrane into motion. As it is common for 


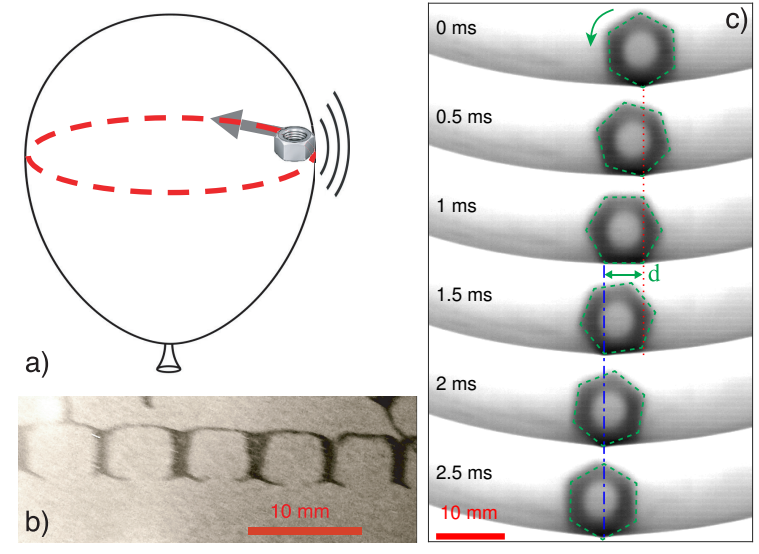

Fig. 1: a) Sketch of the phenomenon. b) Traces left on a transparent balloon by a M6 hex-nut, showing successive impacts of the edges on the membrane. c) Image sequence from high speed video showing the rolling without slipping motion decomposed in time. The red and blue dashed lines indicate the locations of two successive ridges impacts.

drum instruments, the entire membrane vibrates and the deformation is not limited to the vicinity of the nut. However, we demonstrate here that the frequency of the sound emission follows the rate of successive impacts of the spinning object on the membrane, contrary to the classical musical drums and similarly to the washboard or the guiro precussions. This article is organized as follows. The frequency spectrum of the sound is investigated and the physical origin of the dominant frequency, of its overtones and sub-harmonics is elucidated. The influence of the tension within the membrane on the sound intensity is examined and an energetic approach of the acoustic emission is proposed.

Spectral properties of the sound emitted by the screaming balloon. - In this paper, transparent latex balloons(Transparent Size 9 latex balloons, Bishara) were used as they allow one to visualize and track the motion of the nut or coin. These balloons conveniently remain spherical over a large range of volumes. In order to avoid condensation of water inside, the balloons were inflated with dry compressed air. The microphone used for the investigation of the sound spectral characteristics (Microphone of an Apple iPhone 5c) has a bandwidth corresponding to the audible range $(100 \mathrm{~Hz}-20 \mathrm{kHz})$ and acoustic signals are aquired at $24 \mathrm{kHz}$. In order to track the motion of the nut/coin, the balloons were filmed at 300 FPS (Ximea xiQ model MQ013MG-ON) and high-speed videos were recorded up to 10,000 FPS (Phantom - v2511).

Fundamental frequency of the sound. Fig. 2a) shows a typical spectrum of the sound emitted by a hex nut (M5, steel). A clear dominant frequency is visible $\left(f_{0} \simeq 1320\right.$ $\mathrm{Hz}$ in this case). Higher commensurable harmonics, with decaying amplitude, are also clearly visible and are due to the anharmonicity of the signal. Indeed, the nature of the
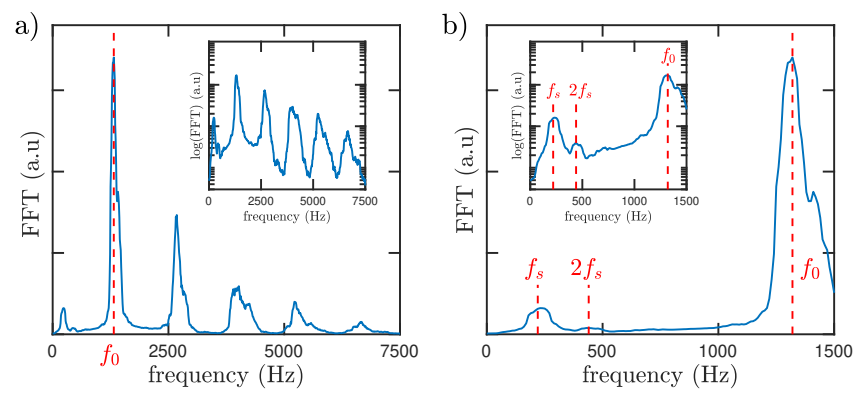

Fig. 2: Fourier transform of a typical acoustic signal emitted by a hex nut spinning in a balloon (a: entire frequency range, b: low frequencies). The insets show the data on a semi-log scale.

impacts is expected to create a non-sinusoidal waveform and their Fourier transform contains multiple frequencies. The resonances observed in Fig. 2 are somewhat broad due to the strong dissipation within the rubber balloon a typical quality factor of 20 was measured and presented in the Supplementary Fig. 2. In order to study the dependence of $f_{0}$ on the relevant parameters, a nut/coin is manually given a high initial spin. The balloon is then firmly held from the top and the bottom as the nut/coin rolls and gradually loses its kinetic energy while emitting an acoustic wave (see supplementary video, parts 1 and 5). As a side note, the sound emitted while the balloon is held down does not significantly differ from that of a free balloon. No assumptions are made regarding the trajectory of the nut/coin (elliptical, helical, horizontal) and its linear velocity, $v$, is computed from particle tracking (at 300 FPS). Measurements were performed for a variety of hex nuts (M2 and M8) made of steel (density $\simeq 8$ ) or nylon (density $\simeq 1.24$ ) as well as using several ridged-edge coins, whose characteristics are given in the Supplementary Table 1. For hex nuts, $d$ is the length of one face (i.e. the distance between two edges), while for coins, $d$ is the distance between two consecutive ridges. The dominant frequency was measured from the power spectrum with an accuracy given by the width of the distribution, and the uncertainties on the velocity $v$ due to the particle tracking algorithm were estimated to remain below $5 \%$.

Fig. 3 displays the dominant frequency, $f_{0}$, as a function of the velocity-to-step ratio, $v / d$ and shows that the two quantities are identical within experimental uncertainties. This holds for all nut/coin size, material, mass as well as for any value of the volume (and hence overpressure inside) of the balloon, and for frequencies ranging from $100 \mathrm{~Hz}$ to almost $10 \mathrm{kHz}$. Unlike drum instruments whose pitch depends on the tension in the membrane, the pitch is clearly only determined by the linear velocity $v$ of the nut/coin along its trajectory, and by the step $d$ of the ridged exciter, regardless of any other quantity:

$$
f_{0}=\frac{v}{d}
$$




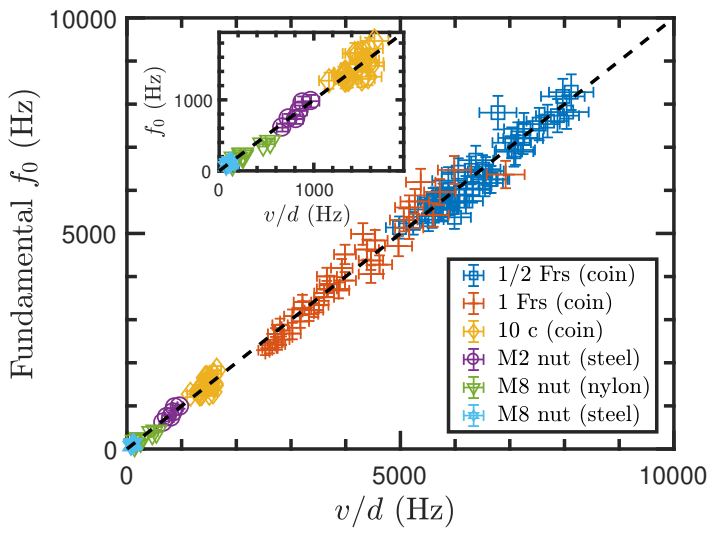

Fig. 3: Dominant frequency of the sound as a function of the speed-to-step ratio $(v / d)$ for a variety of hex nuts and ridgededge coins. The inset is a zoom of the same data set at lower frequencies.

This demonstrates that the nut/coin undergoes a rollingwithout-slipping motion on the membrane (caused by the high friction coefficient of rubber [21]), as also clearly shown in the time lapse sequence of Fig. 1c). Indeed, between two consecutive impacts, the nut travels a distance $d$ and the resulting frequency of impacts is given by $v / d$. These results rule out the relevance of other phenomena as a potential source of the emitted sound, such as any acoustic or mechanical resonance within the balloon (which can still exist, see Supplementary Fig. 1, although it does not set the frequency of the sound) or any hypothetical stickslip motion (as in bowed strings).

Similarly to the washboard or the guiro percussions, the pitch depends linearly on the velocity $v$ of the impacting object (the stick for the percussions and the nut for the screaming balloon) - there is however an extra degree of freedom for the screaming balloon. While, for washboards and guiros, the pitch of the sound is solely given by the velocity of the stick impacting the ridged structure, of step $d$, which radiates the sound, it is set here by the velocity and the geometrical properties of the impacting solid (i.e. the step $d$ of the nut or the coin), independently of the balloon which radiates the sound.

Sub-harmonics. A close examination of the frequency spectrum reveals that it also contains lower harmonics (see Fig. 2). The frequency of the highest amplitude lower harmonic, $f_{s}$ is equal to one sixth of the fundamental frequency $f_{0}\left(f_{s} \simeq 220 \mathrm{~Hz}\right.$ in this case $)$. The ratio $f_{0} / f_{s}=6$ was confirmed using a M2 hex nut for various values of the fundamental frequency, obtained for various velocities and balloon sizes (see $n=6$, orange points in Fig. 4a). This indicates again that the frequency content of the signal depends solely of the step, $d$, and the velocity, $v$, and is independent of the other specification of the nut (mass or diameter) and balloon (Young's modulus, thickness, diameter, pressure). The ratio $f_{0} / f_{s}=6$ for a hexagonal nut indicates that the sub-harmonics are caused by small
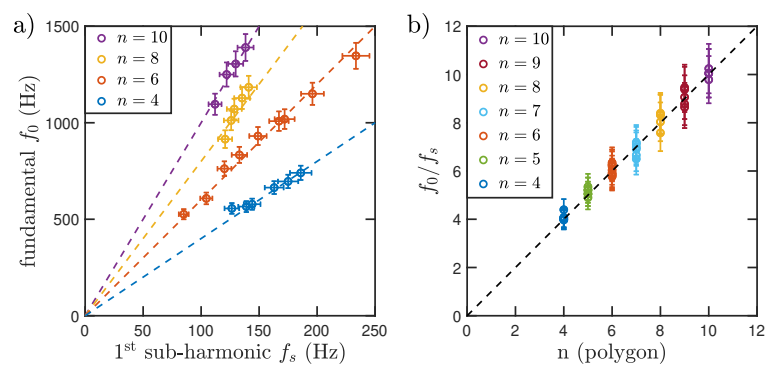

Fig. 4: Fundamental $f_{0}$ versus first sub-harmonic for a variety of regular $n$-polygonal nuts, from squares $(n=4)$ to decagons $(n=10)$. a) $f_{0}$ vs. $f_{s}$ showing a linear dependence of slope $n$ (dashed lines). b) Frequency ratio $f_{0} / f_{s}$ as a function of the number of sides of the polygonal nuts.

asymmetries of the nut. Indeed, if one side of the nut is heavier or lighter, or if an edge is sharper of smoother than others, then the amplitude of the corresponding impact would differ, superimposing a 6-times-smaller frequency to the signal. Thus, the low frequency content of the spectrum does not result from non-linear sub-harmonics generation.

The same procedure was repeated for 3D-printed polygonal nuts, from squares $(n=4)$ to decagons $(n=10)$ (of typical size $10 \mathrm{~mm}$ ). The results are shown in Fig. 4a) where the dashed lines correspond to $f_{0}=n f_{s}$. The same data is plotted in Fig. $4 \mathrm{~b})$ as the ratio $f_{s} / f_{0}$ versus the number of sides of the polygonal nut $n$. Within experimental uncertainties, the sub-harmonic is clearly given by $f_{0} / n$. It is also worth mentioning that sub-harmonics are also visible in the Fourier spectrum of the sound produced by coins (see Fig. 7) but due to the larger number of ridges, $f_{s}$ is considerably lower than $f_{0}$ and for clarity, the data is not shown in Fig. 4.

Sound intensity. - We have shown that the fundamental frequency (along with the overtones and undertones) is given by the rate of collisions and does not directly depend on the mass and material of the nut, or on the exact properties of the balloon. However, these physical quantities are expected to have a clear effect on the sound intensity (i.e. level or power). More specifically, the tension $T$ within the elastic membrane might have a strong effect, as it is the case in all string and drum instruments [1]

The sound intensity was measured using a commercial sound level meter (TES-1350A, TES Corp.) placed $1 \mathrm{~m}$ away (horizontally) from a balloon in which a hex spins around the equator. The sound level was recorded for several balloon sizes (up to $44 \mathrm{~cm}$ in diameter, corresponding to a volume of $45 \mathrm{~L}$ ) for a M5 steel hex nut producing an acoustic wave at $f_{0}=750 \pm 50 \mathrm{~Hz}$, leading to an uncertainty of $\pm 1 \mathrm{~dB}$. The results are shown in Fig. 5c). The sound level is indeed greater (and reaches $65 \mathrm{~dB}$ ) for large volumes but the data shows a counter-intuitive plateau for 

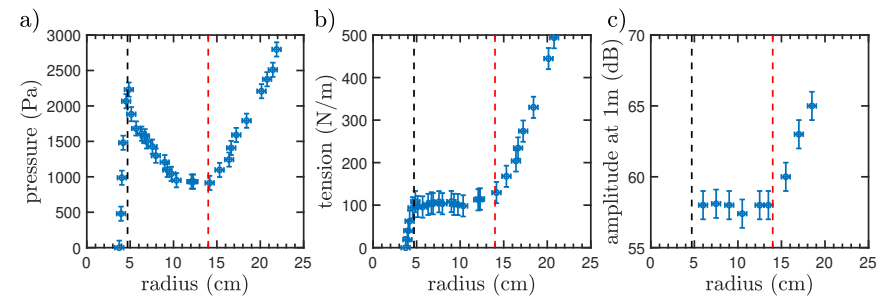

Fig. 5: a) Overpressure inside the balloon, b) tension in the membrane and c) sound amplitude of the acoustic wave emitted by a M5 nut at $f_{0} \simeq 750 \mathrm{~Hz}$, as a function of balloon radius.

small volumes. For an extended range of radii between 6 $\mathrm{cm}$ and $14 \mathrm{~cm}$ (see dashed lines), the acoustic intensity remains constant (around $55 \mathrm{~dB}$ ) although the volume of the balloons increases by a factor 10 (from $0.9 \mathrm{~L}$ to 11.5 $\mathrm{L})$.

In order to elucidate the origin of this plateau, the overpressure, $\Delta P$, in the balloon was measured using a U-tube manometer (with an accuracy of $100 \mathrm{~Pa}$ ) as its volume was varied. Fig. 5a) shows $\Delta P$ as a function of the balloon radius and three classical regimes [22-24] can be observed: an initial sharp increase (radius $\geq 6 \mathrm{~cm}$ ), followed by a slow drop (between $6 \mathrm{~cm}$ and $14 \mathrm{~cm}$ ), and finally a slow increase for larger volumes (radius $\geq 14 \mathrm{~cm}$ ). The intermediate regime is reminiscent of Young-Laplace equations for bubbles and droplets governed by surface tension [25]. For an elastic material however, there exist two limitations: at high deformations the elastomer molecules are over-stretched and the material stiffens [26], leading to an increase in $\Delta P$, whereas at small deformations the material recoils to its original length with vanishing tension.

For a sphere of radius $R$, the mechanical equilibrium imposes $\Delta P=2 T / R$, where $T$ is the tension within the membrane. Note that this equation holds for any spherical membrane and that no assumptions are made regarding $T$. In particular, $T$ is not a priori expected to be a constant (as it would be for a gas/liquid interface) but in general, it should depend on the radius of the sphere, since the elastic material is stretched as the volume of the balloon varies. The membrane tension is shown in Fig. 5b) which reveals that $T$ remains constant for $6 \mathrm{~cm} \leq R \leq 14 \mathrm{~cm}$, which precisely corresponds to the range of constant sound level. Therefore, the plateau in the sound level can be attributed to an unexpected regime in which the tension within the membrane remains constant while the balloon is stretched by a factor 3 .

Conversion of kinetic to acoustic energy. - Evidently, the membrane serves as an amplifier for the sound but the acoustic energy primarily originates from the kinetic energy of the object spinning in the balloon. The kinetic energy $K$ (including the rotational energy) of a ridged-edge solid of mass $m$, rolling without slipping at a velocity $v$, and emitting a sound at a frequency $f_{0}$ reads
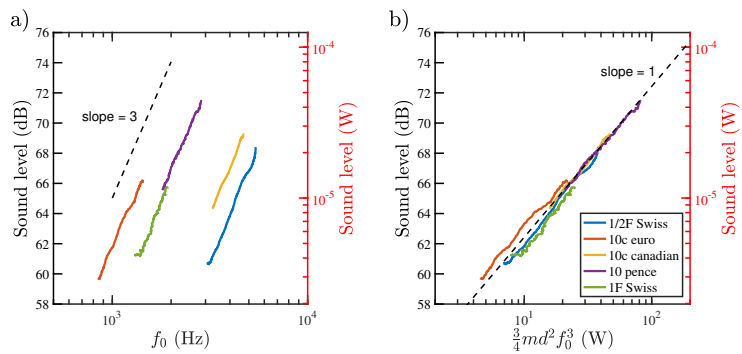

Fig. 6: Sound level (in $\mathrm{dB}$ at $1 \mathrm{~m}$, and in Watts) as a function of the fundamental frequency for a series of coin (a). The same data rescaled as a function of $\frac{3}{4} m d^{2} f_{0}^{3}(\mathrm{~b})$.

$K=3 / 4 m v^{2}=3 / 4 m d^{2} f_{0}^{2}$. Every time a ridge impacts on the membrane (at a rate $f_{0}$ ), a fraction, $\alpha$, of this kinetic energy is converted into acoustic energy. The acoustic power (or sound level), must therefore follow:

$$
\mathcal{P}_{a c}=\alpha \times 3 / 4 m d^{2} f_{0}^{3}
$$

For the study of the frequency dependence, coins are preferably used over hex nut as they allow one to explore a wider range of frequencies and emit a sound far above the natural resonant modes of the balloon (see Supplementary Fig. 1). Indeed, for frequencies corresponding to the first resonant modes of the balloon, the sound intensity can typically increase by a factor 2 . Note however that due to the rapid frequency sweep (due to the velocity decrease) and oscillations (due to non-perfectly horizontal coin/nut trajectory) along with the quality factor of the membrane ( $Q=20$, see Supplementary material), the eigenmodes of the balloon are hardly excited. This justifies the assumption that the conversion factor (or efficiency) $\alpha$ is taken to be a constant. Again, in the low frequency range (0-500 $\mathrm{Hz}$ ), this assumption might not hold as the eigenmodes might resonate, but the experimental data shown in Fig. 6 and 7 clearly show that for higher frequencies $(i 500 \mathrm{~Hz})$ the fraction of kinetic energy converted to acoustics remains constant. Fig. 6 shows the acoustic power as a function of the frequency on a log-log scale for a variety of coins. The sound level in $\mathrm{dB}$ (recorded $1 \mathrm{~m}$ away from a balloon of radius $10 \mathrm{~cm}$ ) was converted into acoustic power (in Watts) assuming a spherical waveform (right $y$ axis). For clarity, the experimental uncertainties are not shown but are of the order of $1 \mathrm{~dB}$. The first conclusion that can be drawn from Fig. 6 a is that the predicted $3^{r d}$ power-law for the frequency dependence is valid for all coins, and that, for a given frequency, the sound intensity is higher for larger $d$. Interestingly, all data sets collapse on a unique master curve when plotted as a function of $m d^{2} f_{0}^{3}$ in Fig. 6 b) that validates (2). This allows one to measure the energy ratio $\alpha=(5.6 \pm 0.5) \cdot 10^{-7}$. This very small value indicates that only a slight fraction of the available energy is converted into an acoustic emission at every impact.

It would be however more relevant to compare the acoustic power to the loss of energy per impact (rather 


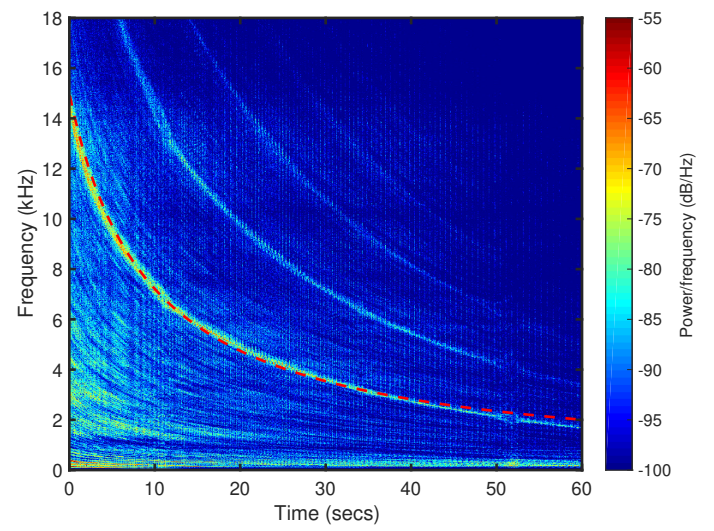

Fig. 7: Spectrogram of the sound emitted by a coin $(1 / 2$ Swiss franc). Dashed line: theoretical prediction using Eq. 3.

than to the absolute value of the energy). Fig. 7 shows the spectrogram obtained with a coin, initially emitting a sound at $f_{0}(t=0)=15 \mathrm{kHz}$ (as a side note the overtones and sub-harmonics are clearly visible). As its initial energy is slowly converted into acoustic energy as well as dissipated in the membrane, its velocity decreases along with the emitted frequency. Assuming that a fraction $\beta$ of the kinetic energy is dissipated at every impact, one can find the time-dependence of the fundamental frequency (see Supplementary, part IV):

$$
f_{0}(t)=\frac{f_{0}(t=0)}{1+\beta t f_{0}(t=0) / 2}
$$

The red dashed line shows a fit $\left(\beta=14 \cdot 2 \cdot 10^{-6}\right)$ of the data using this prediction, and the very good agreement justifies the assumption that the relative energy loss per impact, $\beta$, is constant. This small value explains the long duration of a typical run: it takes $\tau=2 /\left(\beta f_{0}(t=0)\right) \simeq$ $9.25 \mathrm{~s}$ for the frequency to be halved (see supplementary video part 5). The ratio $\alpha / \beta \simeq 4 \%$ appears to be the relevant parameter to characterize the efficiency of the energy conversion: only $4 \%$ of the energy actually available is used to emit sound while the rest is dissipated (in all likelihood in the membrane). The high damping in the membrane is consistent with the low quality factor of the free oscillations of the balloons, $Q \simeq 20$, (see Supplementary Fig. 2), which indicates that most of the energy dissipates within a few vibrations. However, this seemingly low efficiency is in reality rather high for a drum instrument or an electro-acoustic loudspeaker [27].

Let us finally mention that the energy conversion of hex nuts (whose edges are more prominent than coins) is considerably higher, leading to a louder sound (typically $+30 \mathrm{~dB}$ at any given frequency), associated with a faster decay rate (no more than a few seconds vs. a few minutes for coins, see supplementary video).
Conclusion. - We have shown that the fundamental frequency of the screaming balloon is given solely by the rate of successive impacts of the edges of a nut/coin rolling without sliding on the membrane. The richness of the spectrum is due to the anharmonicity of the signal (for higher harmonics) and to irregularities in the shape of the nut (for sub-harmonics). The tension within the membrane was found to remain constant over a wide range of radii, which explains the plateau in the acoustic intensity of the sound when the volume of the balloon is increased. Finally we showed that the acoustic power is directly related to the kinetic energy of the nut/coin.

One aspect of the sound would probably deserve further investigation: as nuts/coins remain close in general to the equator, the emitted sound might show a pronounced anisotropy. Moreover, a detailed analysis of the effect of the shape of the ridges on a coin (whether sharp or smooth) could shed some light on the conversion ratio from kinetic to acoustic energy. Furthermore, a study of the interplay between the frequency imposed by the nut/coin and the resonant modes of the balloon itself would probably be interesting. Finally, in our study, the nut/coin is given an initial velocity by manually spinning or shaking the balloon but mechanisms by which the nut/coin is set into motion was not explored.

Acknowledgments. - This work was supported by the École Normale Supérieure de Lyon and University Claude Bernard, Lyon, France. The use of the high speed camera Phantom v2511 was made possible thanks to financial support from CNRS INP. The authors are grateful to A. Marquet, C. Pacary, C. Praud, H. Remise-Charlot and C. Rouvière for fruitful discussions.

\section{REFERENCES}

[1] Fletcher N. H. and Rossing T. D., The Physics of Musical Instruments 2nd Edition (Springer-Verlag New York Inc.) 2010.

[2] Fletcher N. H., Rep. Prog. Phys., 62 (1999) 723-764.

[3] Cremer L., The physics of the violin (MIT press Cambridge) 1984 .

[4] Woodhouse J., Rep. Prog. Phys.77, 2014 (115901) .

[5] Benade A. H., Scientific American, 229 (1973) 24.

[6] Olson H. F., Music, physics and engineering Vol. 1769 (Courier Corporation) 1967.

[7] Worland R., J. Acoust. Soc. Am., 139 (2016) 2011.

[8] Sсотt J. F. M. and Woodhouse J., Philos. T. R. Soc. Lond., 339 (1992) 587.

[9] Rayleigh L., The London, Edinburgh, and Dublin Philosophical Magazine and Journal of Science, 22 (1911) 225.

[10] Raman C. V., Proc. Indian Acad. Sci.A, 1 (1935) 179.

[11] Rossing T. D., J. Acoust. Soc. Am., 95 (1994).

[12] Courtois M., Guirao B. and E. F., Eur. J. Phys., 29 (2008) .

[13] Arbel L., Schechner Y. Y. and Amir N., Appl. Acoust., 124 (2017) 1. 
[14] Terwagne D. and Bush J. W. M., Nonlinearity, 24 (2011) R51.

[15] Akay A., J. Acoust. Soc. Am., 111 (2002) 1525.

[16] Haskell P. T., Insect Sounds 1st Edition (Witherby, London) 1961.

[17] White H. and White D., Physics and Music: The Science of Musical Sound Dover Books on Physics (Dover Publications) 2014.

[18] Schouten J. F., Proc. Kon. Ned. Akad. v. Wetenseh., 43 (1940) 356.

[19] Flanagan J. L. and Guttman N., J. Acoust. Soc. Am., 32 (1960) 1308.

[20] Flanagan J. L. and Guttman N., J. Acoust. Soc. Am., 32 (1960) 1319.

[21] Schallamach A., Wear, 17 (1971) 301.

[22] Needleman A., Int. J. Solids Struct., 13 (1977).

[23] Merrit D. R. and F. W., Am. J. Phys., 46 (1978) .

[24] Muller I. and Struchtrup N., Math. Mech. Solids, 7 (2002) .

[25] Tritton D. J., Physical Fluid Dynamics 1st Edition (Clarendon Press) 1988.

[26] Treloar L. R. G., The Physics of Rubber Elasticity 1st Edition (Oxford University Press) 1975.

[27] MCCARThy B., Sound systems: design and optimization: modern techniques and tools for sound system design and alignment (CRC Press) 2012. 